\title{
JNVESTJGAAJÓN
}

\section{Desarrollo de pasta untable de aceituna variedad Sevillana}

\author{
Por Marco Schwartz ${ }^{a}$ Vilma Quitral ${ }^{\star b}$ Claudia Daccarett $^{a}$ y Julio Callejas ${ }^{a}$
}

\author{
${ }^{a}$ Departamento de Agroindustria. Facultad de Ciencias Agronómicas. Universidad de Chile \\ ${ }^{\text {b }}$ Departamento de Nutrición. Facultad de Medicina. Universidad de Chile. Santiago de Chile \\ ( ${ }^{\star}$ Autor para la correspondencia: vquitral @ med.uchile.cl)
}

\section{RESUMEN} \section{villana}

Desarrollo de pasta untable de aceituna variedad Se-

Este estudio consistió en desarrollar una pasta untable de aceituna, variedad verde Sevillana o Gordal. La elaboración de la pasta consistió en deshuesar las aceitunas, triturarlas y adicionarles conservantes (sorbato de potasio y benzoato de sodio) y antioxidante (T. B. H. Q.), finalmente se envasaron. Ésta fue caracterizada microbiológica, físico-química y sensorialmente, y se evaluó su comportamiento durante 3 meses de almacenamiento a temperatura ambiente $\left(18^{\circ} \mathrm{C}\right)$ y en refrigeración $\left(4^{\circ} \mathrm{C}\right)$. El recuento microbiano de la pasta es menor a $100 \mathrm{ufc} / \mathrm{ml}$. Sensorialmente el producto fue aceptado y esta condición no varió durante el almacenamiento, al igual que el contenido de aceite $(70 \%)$ y $\mathrm{a}_{\mathrm{w}}(0,90)$. El pH diminuye con el tiempo, por lo tanto, la acidez aumenta; viéndose afectado por la temperatura de almacenamiento. El índice de peróxido, aumenta en el tiempo (5 a 27 meq peróxido/kg aceite) y es mayor en condiciones ambientales, sin embargo, no es percibido como variación en la rancidez sensorial. En cuanto al color, el principal cambio producido es un oscurecimiento de la pasta expresado en el parámetro $L^{*}$. La vida útil de la pasta de aceituna alcanza a 90 días, siendo apta su conservación a temperatura ambiente y de refrigeración.

PALABRAS CLAVE: Aceituna - Color - Índice de Peróxido - Microbiológico - Pasta de aceituna - Pulpa de aceituna - Sensorial.

\section{SUMMARY}

Development of spreadable olive paste from the Sevillana variety

The development of a spreadable olive paste was carried out. Sevillian or Gordal green olive varieties were used in the paste. Pastes were prepared by crushing olives after pit removal and adding preservatives (potassium solvate and sodium benzoate), and antioxidants (T. B. H. Q.) to crushed olives. These were characterized by recording microbial, physical, chemical and sensory measurements and shelf life was evaluated over three months of storage at room temperature $\left(18^{\circ} \mathrm{C}\right)$ and under refrigeration $\left(4^{\circ} \mathrm{C}\right)$. The final microbial count of the paste was less than $100 \mathrm{ufc} / \mathrm{ml}$. Sensory testing demonstrated that it was acceptable and storage conditions did not significantly affect the product. The $\mathrm{pH}$ of the paste decreased with time (4.3 to 4.17$)$, resulting in increased acidity which was affected by storage temperature. The peroxide index, increased with time, (from 5 to 27 meq peroxide/kg oil). However, the pastes were not perceived to be rancid after sensory testing. Regarding the color, the main change observed was a darkening of the paste, as determined using the parameter $L^{*}$. The shelf life of the olive paste was 90 days either under refrigeration or at room temperature.

KEY-WORDS: Color - Microbiological - Olive - Olive paste-Olive pulp-Peroxide value - Sensory.

\section{INTRODUCCIÓN}

Los beneficios para la salud del consumo de aceitunas y sus derivados se deben a la presencia del ácido oleico (C18:1) (Berry et al., 1992; Mata et al., 1997; Hargrove et al., 2001) y a otras sustancias biológicas que incluyen tocoferoles, polifenoles y fitoesteroles, los que poseen propiedades antioxidantes y antiinflamatorias (Martinez De Victoria y Mañas, 1998; Jacotot, 2001; Calíbrese, 2002; Conforti et al., 2009; Acín et al., 2007).

Los productos untables como margarina, mantequilla, queso crema, paté, etc. son de gran consumo en Chile, tanto para acompañamiento de comidas como para untar en pan, muchos de ellos con alto contenido de colesterol (Scmidt-Hebbel et al., 1992; USDA, Database). Por lo que se hace necesario disponer de un alimento en forma untable que sea libre de colesterol y que resulte beneficioso para la salud. Una buena opción es recurrir a las olivas o aceitunas como materia prima, fruto que posee componentes beneficiosos para la salud.

La aceituna es el fruto proveniente del olivo (Olea europea), el cual se cosecha en distintos estados de madurez, de acuerdo al producto que se quiera obtener (aceituna verde, "mulata" o negra) y luego es sometido a un proceso industrial para ser consumido finalmente en forma preparada o conservada (Hermoso et al., 1998; FIA, 1999). Las aceitunas verdes corresponden a las cosechadas en el ciclo de maduración antes del invierno; y pueden presentar una coloración que varia entre el verde y el amarillo paja (Fernández et al., 1985; FAO, 1991; Consejo Oleícola Internacional, 2002). Al desglosar el consumo mundial por tipos de aceitunas de mesa, predomina el consumo de verdes con 
un $42 \%$, en tanto el consumo de negras y de color cambiante, representan el $36 \%$ y el $22 \%$, respectivamente (FIA, 1999).

En Chile, el olivo se encuentra distribuido desde la $1^{\circ}$ a la $8^{\circ}$ región, y también en algunos microclimas de la $9^{\circ}$ región (FIA, 2002) ocupando una superficie plantada al año 2004 de 6.000 hectáreas, de las cuales aproximadamente el $30 \%$ se concentra en la $3^{\circ}$ región (ODEPA, 2009); principalmente en las comunas de Freirina y Huasco. En esta última, la producción olivícola está dirigida mayormente a la aceituna de mesa, siendo Sevillana la variedad predominante $(80 \%$ de la superficie) (Callejas y Pastene, 2000). Dado su bajo contenido en aceite, la producción de la variedad Sevillana se destina principalmente al mercado de aceituna de mesa. En el año 2007 se produjeron 14.000 toneladas de la variedad Sevillana (Prochile, 2009). Del total de producción destinada a la comercialización, alrededor del 10 al $15 \%$ de los frutos se rechazan en la industria por presentar manchas, golpes o estar rallados. Estas aceitunas se clasifican bajo la denominación de "aceitunas de rechazo" (Callejas et al., 2001), y son éstas las que seria interesante procesar para que no constituyan una pérdida.

La tendencia general observada en todos los países, es hacia un producto olivícola más sofisticado, esto significa un retroceso de las aceitunas enteras y un incremento de productos de aceitunas con valor agregado (Schwartz et al., 2001; FIA,
2002). Por lo anterior, la utilización de los frutos de aceitunas no exportable en el desarrollo de un producto como la pasta untable de aceituna, permite poner a disposición del mercado un alimento con las propiedades saludables del aceite de oliva y funcional a la dieta mediterránea.

Por lo anteriormente expuesto, el objetivo de este estudio fue desarrollar pasta untable de aceituna de mesa de la variedad sevillana (equivalente a la variedad Gordal en España), caracterizándola desde el punto de vista microbiológico, físico-químico y sensorial, además, evaluar su comportamiento durante tres meses de almacenamiento a dos temperaturas diferentes.

\section{MATERIALES Y MÉTODOS}

\subsection{Materia prima}

Se utilizaron aceitunas de la variedad Sevillana o Gordal, procesadas al estilo verde sevillano, de descarte de exportación, provenientes de Huasco, Región de Atacama, Chile.

\subsection{Elaboración de la pasta untable}

Se elaboró la pasta untable en base al diagrama de la figura 1. Para el deshuesado de las aceitunas

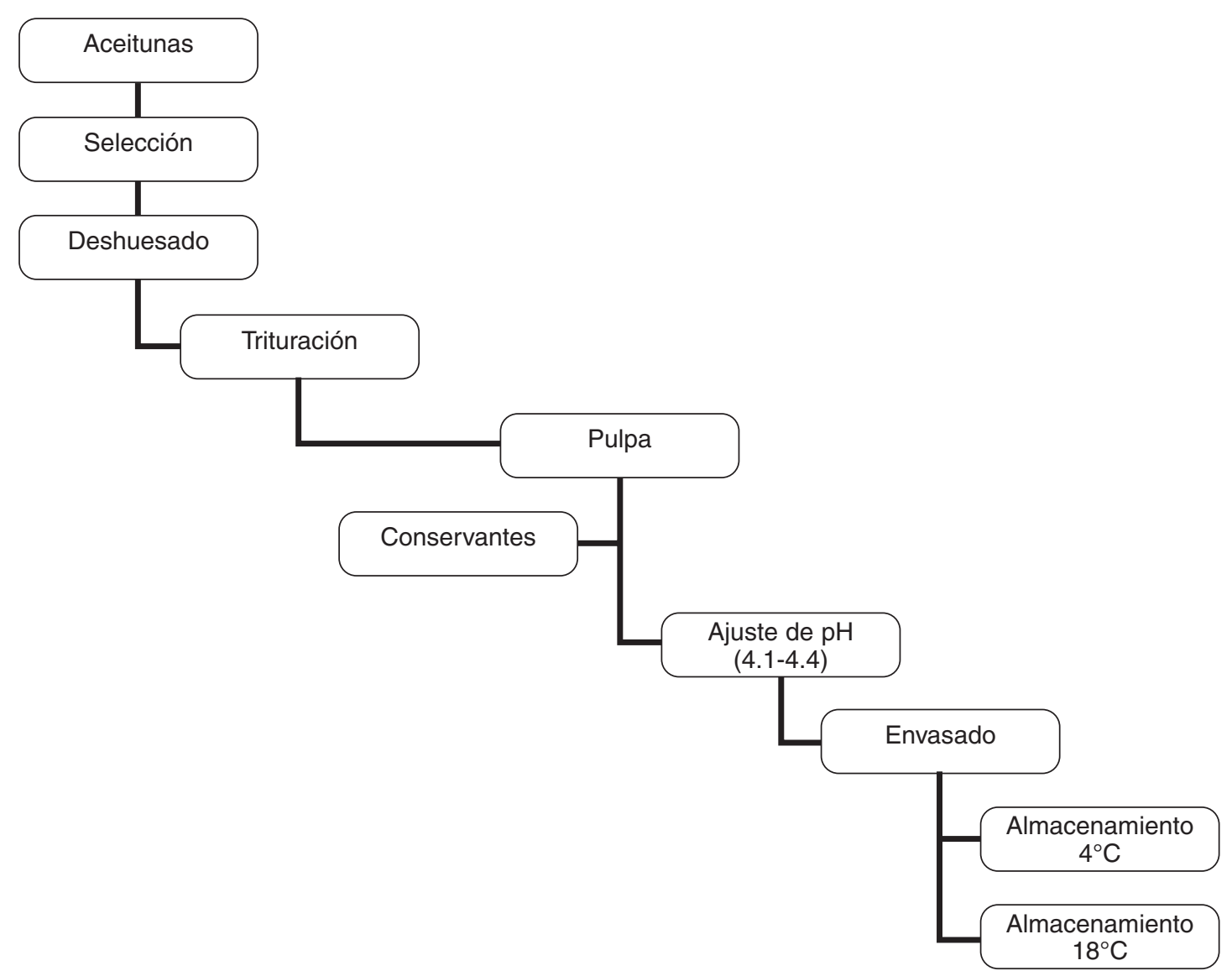

Figura 1

Línea de flujo para la elaboración de pasta untable de aceituna. 
se usó sacabocado de diámetro número 5 , correspondiendo a $9 \mathrm{~mm}$. Para la trituración se utilizó una picadora marca Moulinex, modelo 320. Una vez obtenida la pulpa de adicionó: sorbato de potasio (2 $\mathrm{g} / \mathrm{kg})$, benzoato de sodio $(1 \mathrm{~g} / \mathrm{kg})$ y T.B.H.Q. $(0.2$ $\mathrm{g} / \mathrm{kg}$ ). Para ajustar el $\mathrm{pH}$ se acidificó con ácido láctico. La pasta se envasó en frascos de vidrio de 200 $\mathrm{ml}$ y se almacenaron en refrigeración $\left(4^{\circ} \mathrm{C}\right)$ y a temperatura ambiente $\left(18^{\circ} \mathrm{C}\right)$

\subsection{Análisis}

\section{Análisis microbiológicos}

El análisis microbiológico aplicado para el recuento de microorganismos aerobios mesófilos viables (R.A.M.) se basó en la NCh2659.of2002. Para el recuento de enterobacterias se aplicó la metodología descrita en NCh2676.of2002 y el recuento de hongos y levaduras se realizó en base NCh2734.of2002

\section{Análisis fisicoquímicos}

El pH ( AOAC, 1995) fue determinado por medición directa con potenciómetro. La acidez titulable (AOAC, 1995) mediante titulación directa de la muestra expresando el resultado en \% de ácido láctico. El Índice de Peróxidos fue determinado por el método de la AOCS (1993), expresando el resultado en miliequivalentes de peróxidos por $\mathrm{kg}$ de aceite. El color fue determinado por un método instrumental evaluando parámetros $L^{*}, a^{*}, b^{*}$ con un colorímetro Minolta CR-200b. El contenido total de aceite por un método gravimétrico con extracción en equipo Soxhlet (AOCS, 1993). La actividad de agua $\left(a_{w}\right)$ fue determinada con un analizador LUFFT modelo 5803.

\section{Análisis sensorial}

Se realizaron dos pruebas:

Aceptabilidad: con panel no entrenado de 43 personas, aplicando una escala no estructurada de 15 puntos desde "me disgusta extremadamente" con valor 0 hasta "me gusta extremadamente" con un valor de 15, pasando por el valor central de indiferencia.

Calidad con escala no estructurada de 15 puntos por parámetro: con panel entrenado de 15 personas en que se evaluó apariencia (desde "disgregada" con valor 0 hasta "compacta" con valor de 15), color, aroma, rancidez, gusto ácido, salado, amargor, consistencia (desde "granulosa" hasta "untable") y sabor (desde "sin sabor" hasta "sabor muy característico") (Wittig, 2001; Anzaldúa-Morales, 1994)

\section{Análisis estadístico}

El diseño experimental para los análisis físicos y químicos fue de estructura factorial $2 \times 4$ ( 2 tempe- raturas de almacenamiento y 4 tiempos de almacenamiento: 0,30 , 60 y 90 días) con tres repeticiones para cada ensayo. El diseño experimental para el análisis sensorial fue en bloques completos al azar con estructura factorial $2 \times 2$ ( 2 temperaturas de almacenamiento dos tiempos de almacenamiento: 0 y 90 días). En ambos casos los resultados se analizaron estadísticamente con Análisis de Varianza (ANDEVA) y prueba de rango múltiple de Duncan, en caso de detectarse diferencias significativas.

\section{RESULTADOS Y DISCUSIÓN}

La relación entre el hueso y la pulpa de las aceitunas utilizadas en este estudio se muestra en la Tabla 1; la pulpa esta constituida por agua $(68,4 \%)$, aceite $(21,6 \%)$ y sólidos totales $(10,0 \%)$; los que a su vez corresponden a $44,5 \%$ de agua, $14,1 \%$ de aceite y $6,5 \%$ de sólidos totales sobre el total del fruto. El contenido de aceite para la variedad Sevillana o Gordal señalado por FAO (1991) es menor $(6,25 \%)$, en tanto el de agua es $73,7 \%$. El rendimiento en pulpa obtenido fue del orden de $61 \%$; el resto está asociado al hueso (34,9\%) y pérdidas en el proceso de elaboración. La relación aceituna/hueso presente en las aceitunas es similar a la que establece FAO (1991) para la variedad sevillana, que es de 3:1.

El análisis microbiológico de bacterias mesófilas aerobias viables en las muestras durante el tiempo de almacenamiento indica un recuento menor de $10 \mathrm{ufc} / \mathrm{ml}$ y solo se produjo un resultado positivo a los 90 días de almacenamiento, con $2,5 \times 10^{2} \mathrm{ufc} / \mathrm{ml}$. De acuerdo al Reglamento Sanitario de Alimentos de Chile (Ministerio de Salud, 1998) acerca de "alimento listo para el consumo", debe tener un máximo de $5 \times 10^{5} \mathrm{ufc} / \mathrm{ml}$. El recuento total en las muestras de pasta de aceituna es mucho menor. Por lo tanto se pueden consumir hasta los 90 días.

El recuento de enterobacterias, hongos y levaduras fue idéntico para las muestras refrigeradas y almacenadas a temperatura ambiente $(<10 \mathrm{ufc} / \mathrm{ml})$ e indican los mismos resultados de carácter sanitario. Gracias a la adición de sorbato de potasio y benzoato de sodio que previenen el desarrollo de mohos en alimentos ácidos (Tortora et al., 1993). El acido benzoico es muy efectivo para la inhibición de mohos y levaduras, pero es menos efectivo con-

Tabla 1

Peso, relación y rendimiento promedio para la obtención de pulpa de aceituna

\begin{tabular}{lc}
\hline \multicolumn{1}{c}{ Parámetro } & Valor \\
\hline Peso aceitunas $(\mathrm{g})$ & 100 \\
Peso huesos $(\mathrm{g})$ & 34.9 \\
Peso pulpa $(\mathrm{g})$ & 61.4 \\
Relación aceituna/hueso & $8.5: 3$ \\
Relación pulpa/hueso & $7: 4$ \\
Coeficiente técnico (kg aceitunas/kg pasta) & 1.61 \\
\hline
\end{tabular}


tra bacterias, mientras que el acido sórbico es un potente inhibidor del crecimiento de una extensa variedad de hongos, levaduras y bacterias (Turanta et al., 1999). Chikthimmah et al., (2003) estudiaron el efecto del benzoato de sodio $(0.05 \%$ peso/volumen) junto al ácido fumárico $(0.15 \%$ peso/volumen) sobre la destrucción de Escherichia coli en sidra de manzana ( $\mathrm{pH}$ cercano a 3.5) inoculada con esta bacteria y almacenada a $5^{\circ} \mathrm{C}$ y encontraron que al cabo de 75 horas la población de esta bacteria presentó una disminución logarítmica de $10^{5}$. Turanta et al., (1999) aplicaron dosis de 500 y 250 ppm de benzoato de sodio y sorbato de potasio respectivamente, en aceitunas negras y lograron inhibir el desarrollo de hongos y levaduras durante el almacenamiento.

Con los resultados microbiológicos obtenidos en la pasta de aceitunas durante el almacenamiento, se puede afirmar que las condiciones de elaboración fueron adecuadas sanitariamente; los conservantes utilizados fueron los apropiados; la acidificación del producto contribuyó en la disminución del desarrollo microbiano; y los envases utilizados fueron lo suficientemente estériles y herméticos, ya que no se presentó contaminación microbiológica.

Las características fisicoquímicas de la pasta de aceituna y el efecto de temperatura y tiempo de almacenamiento, se presentan en la Tabla 2.

$\mathrm{El} \mathrm{pH}$ permite controlar el recuento microbiano de los alimentos, como es el caso de la pasta de aceituna, entre ellos el Clostridium botulinum, que no se puede desarrollar en alimentos cuyo $\mathrm{pH}$ sea inferior a 4,5 (Venegas et al., 1990; Mazzobre et al., 2002). Durante el almacenamiento, se observó una disminución del pH en la pasta de aceituna; esto debido probablemente a la presencia de microorganismos que producen ácido láctico, lo que disminuye el $\mathrm{pH}$. Resultados similares obtuvieron Ünal y Nergiz (2003) al almacenar aceitunas verdes por 12 meses, apreciando una constante disminución de $\mathrm{pH}$. Los valores de $\mathrm{pH}$ de la pasta de aceituna son lo suficientemente bajos como para inhibir la actividad de diversas enzimas presentes en el fruto, como hidrolasas, lipoxigenasas y otras. (Kalua et al., 2007).

El contenido de aceite no presentó variación durante 90 días de almacenamiento en la pasta de aceitunas. La temperatura de almacenamiento no influyó en el contenido de aceite. Gutiérrez et al., (1992) encontraron que al almacenar aceitunas a temperatura ambiente, previo a la extracción de aceite, el rendimiento obtenido era menor que al almacenarlas a $5^{\circ} \mathrm{C}$, ya que los frutos almacenados en condiciones ambientales demostraron desarrollo de pudrición, lo que causa deterioro de la estructura de los tejidos y secreción de fluidos; no obstante, en la pasta de aceituna en estudio no se presentó pudrición, por lo que este efecto no se observa.

El valor de actividad de agua se mantuvo constante en el tiempo y no varió con la temperatura. El valor de 0.90 para la $a_{w}$ de la pasta de aceitunas es alto, lo que favorece el desarrollo microbiano (Eisenberg, 2007), por lo que este alimento logra su estabilidad gracias a su acidez.

Se presenta un aumento significativo del índice de peróxido durante el tiempo de almacenamiento, siendo mayor a los 90 días, con un valor de 24.69 meq. peróxido/kg aceite. Además, la temperatura de conservación tiene influencia en el grado de rancidez que presenta la pasta de aceituna, siendo mayor el índice de peróxido en condiciones ambientales. García et al., (1996a y 1996b) encontraron que al almacenar las aceitunas a $5^{\circ} \mathrm{C}$ por 60 días, previo a la extracción del aceite, éste contenía un menor grado de oxidación (determinado por medio del índice de peróxido) que si las aceitunas eran almacenadas a temperatura ambiente. Según Kalua et al., (2008) almacenamiento de aceitunas a temperaturas de refrigeración $\left(0-8^{\circ} \mathrm{C}\right)$ y atmósfera modificada mantienen la calidad y la prolongan durante el almacenamiento. Las temperaturas más

Tabla 2

Efecto del tiempo y temperatura de almacenamiento sobre las características físico-químicas de pasta de aceituna

\begin{tabular}{|c|c|c|c|c|c|c|}
\hline \multirow{2}{*}{ Determinaciones analíticas } & \multicolumn{4}{|c|}{ Factor tiempo } & \multicolumn{2}{|c|}{ Factor temperatura } \\
\hline & 0 & 30 & 60 & 90 & $18^{\circ} \mathrm{C}$ & $4^{\circ} \mathrm{C}$ \\
\hline $\mathrm{pH}$ & $4,30^{b}$ & $4,39^{\mathrm{a}}$ & $4,16^{c}$ & $4,17^{c}$ & $4,27^{a}$ & $4,24^{a}$ \\
\hline Acidez (\% ác. Láctico) & $0,43^{a}$ & $0,41^{a}$ & $0,41^{\mathrm{a}}$ & $0,43^{a}$ & $0,43^{a}$ & $0,41^{\mathrm{a}}$ \\
\hline Contenido de aceite(\%) & $70,85^{a}$ & $72,07^{a}$ & $7214^{a}$ & $71,24^{a}$ & $71,35^{\mathrm{a}}$ & $71,80^{\mathrm{a}}$ \\
\hline $\begin{array}{l}\text { aw } \\
\text { Índice de peróxido }\end{array}$ & $0,90^{\mathrm{a}}$ & $0,90^{\mathrm{a}}$ & $0,91^{a}$ & $0,90^{\mathrm{a}}$ & $0,90^{\mathrm{a}}$ & $0,90^{\mathrm{a}}$ \\
\hline (meq Peróxido/Kg aceite) & $5,44^{\mathrm{c}}$ & $13,25^{\mathrm{b}}$ & $13,70^{a}$ & $24,69^{a}$ & $17,18^{a}$ & $11,36^{b}$ \\
\hline$L^{*}$ & $51,37^{\mathrm{a}}$ & $50,07^{b}$ & $51,17^{\mathrm{a}}$ & $49,43^{b}$ & $50,83^{a}$ & $50,19^{b}$ \\
\hline$A^{*}$ & $-1,35^{b}$ & $-1,37^{b}$ & $-0,95^{a}$ & $-1,32^{b}$ & $-1,30^{\mathrm{a}}$ & $-1,19^{a}$ \\
\hline \multirow[t]{3}{*}{ Color } & $23,15^{b}$ & $23,10^{b}$ & $23,90^{\mathrm{a}}$ & $22,60^{b}$ & $23,42^{a}$ & $22,96^{b}$ \\
\hline & $93,34^{a}$ & $93,39^{a}$ & $92,26^{b}$ & $93,33^{a}$ & $93,19^{a}$ & $92,98^{a}$ \\
\hline & $23,19^{b}$ & $23,14^{b}$ & $23,92^{a}$ & $22,64^{b}$ & $23,45^{a}$ & $22,99^{b}$ \\
\hline
\end{tabular}

Letras distintas indican diferencia significativa con error de 5\%.

Letras minúsculas comparan los diferentes tiempos de almacenamiento; letras mayúsculas comparan las distintas temperaturas de almacenamiento, siempre en forma horizontal (determinación analítica). 
usadas son $3-5^{\circ} \mathrm{C}$, así se reduce la proporción de las reacciones químicas y la actividad microbiana.

El índice de peróxido y la acidez son útiles índices de control de calidad de alimentos. Los valores límites que se han establecido en Japón son de no más de $30 \mathrm{meq} / \mathrm{kg}$ y 3 respectivamente, estos valores se han elegido porque ellos indican el estado inicial del deterioro de grasas y aceites (Gotoh \& Wada, 2006). De acuerdo a estos valores, la pasta no alcanza el deterioro en los 90 días de almacenamiento.

Cuando las aceitunas son microbiológicamente estables y tienen un $\mathrm{pH}$ bajo, el modo de determinar su vida útil es por medio del color (Sánchez et al., 1997). Asimismo, este parámetro puede verse afectado por condiciones extremas; por ejemplo, García et al., (1999) señalan que cuando las aceitunas almacenadas poseen un $\mathrm{pH}$ inferior a 6,5 pueden sufrir decoloración y pardeamiento.

Al término del almacenamiento, disminuyó la luminosidad $\left(L^{*}\right)$, este resultado se contradice con lo que señalan Sánchez et al., (1997) quienes encontraron que al almacenar aceitunas de mesa en oscuridad no afecta su luminosidad, expresada en el parámetro $L^{*}$; sin embargo, esto sí sucede en presencia de luz. Por otro lado, García et al., (1999) encontraron que aceitunas $(\mathrm{pH}: 4,3)$ almacenadas a $25{ }^{\circ} \mathrm{C}$ presentan oscurecimiento de su superficie, posiblemente por la oxidación de los polifenoles. La pasta de aceitunas resultó significativamente más clara a temperatura ambiente que en refrigeración.

La cromaticidad rojo - verde $\left(a^{*}\right)$ (rojo para los valores positivos y verde para los negativos) no varia con el tiempo ni la temperatura de almacenamiento en la pasta de aceituna. La cromaticidad amarillo - azul ( $b^{\star}$ ) (amarillo para valores positivos y azul, para los negativos) solo se ve afectada a los 60 días de almacenamiento, presentando un valor más alto, es decir más amarillo. La temperatura también afecta este parámetro, desarrollándose mas el color amarillo a temperatura ambiente. El tono $\left(H^{*}\right)$, que expresa la relación entre $a^{*} y b^{*}$ (verde y amarillo), no varia en la pasta de aceituna. La saturación del color $\left(\mathrm{C}^{*}\right)$ se mantiene sin variación. La temperatura de conservación influye en las pas- tas de aceituna natural, siendo mayor a temperatura ambiente.

Los resultados del análisis sensorial obtenidos muestran que en la pasta de aceituna, el tiempo y temperatura de almacenamiento, no afectan la aceptabilidad ni la calidad. La aceptabilidad (medida en escala no estructurada de 15 puntos) no presenta diferencia significativa entre los tiempos de almacenamiento (9.33 y 10.77 a 0 y 90 días respectivamente) ni entre las temperaturas (10.10 y 10.00 para $4^{\circ}$ y $18{ }^{\circ} \mathrm{C}$ respectivamente), lo que es beneficioso ya que indica que la pasta mantiene la misma aceptabilidad a través del tiempo y que se puede almacenar a temperatura ambiente y/o refrigerada.

Respecto a la calidad sensorial de la pasta de aceituna natural, se consideró de buena apariencia; color, aroma, gusto ácido y amargor normales; con leve sabor a rancio; salada; de textura y sabor agradable. (Tabla 3)

La pasta de aceituna en estudio, fue calificada con un alto nivel de salado -que se puede disminuir si es necesario-, esto debido a que las aceitunas utilizadas en la elaboración de la pasta contenían un $4 \%$ de sal inicialmente. El gusto amargo de las aceitunas se atribuye a compuestos fenólicos presentes en ella, principalmente a oleuropeína (Bouaziz et al., 2004). Marsillo (2002), establece que la percepción de dicho gusto se puede reducir en presencia de cloruro sódico, y tal vez, es por ello que se consideró como normal el amargor de pasta de aceitunas, enmascarado tras un salado excesivo. La rancidez se consideró ligera, sin presentar diferencias al finalizar el almacenamiento; no obstante, los índices de peróxido aumentaron significativamente al cabo de tres meses. El color de las aceitunas verdes al estilo sevillano ( $y$, por lo tanto, de la pasta de aceituna en estudio) tiende al amarillo pajizo, probablemente por el efecto de la degradación de los pigmentos clorofílicos en medio ácido; el color se consideró normal.

Al cabo de tres meses de almacenamiento, la pasta de aceituna natural refrigerada es más oscura que la almacenada a temperatura ambiente, lo que coincide con la medición del color instrumental

Tabla 3

Efecto del tiempo y temperatura de almacenamiento sobre la calidad sensorial de pasta de aceituna

\begin{tabular}{lccrr}
\hline \multirow{2}{*}{ Calidad sensorial: Parámetros } & \multicolumn{2}{c}{ Factor tiempo (días) } & \multicolumn{2}{c}{ Factor temperatura $\left({ }^{\circ} \mathbf{C}\right)$} \\
\cline { 2 - 5 } & \multicolumn{1}{c}{$\mathbf{0}$} & $\mathbf{9 0}$ & $\mathbf{1 8}{ }^{\circ} \mathbf{C}$ & $\mathbf{4}^{\circ} \mathbf{C}$ \\
\hline Apariencia & 10.13 & 9.84 & 9.68 & 10.30 \\
Color & 8.24 & 7.70 & 8.08 & 7.85 \\
Aroma & 8.66 & 7.57 & 8.14 & 8.09 \\
Rancidez & 3.67 & 3.12 & 2.73 & 4.06 \\
Gusto ácido & 7.38 & 8.47 & 7.84 & 8.01 \\
Salado & 10.23 & 11.15 & 7.93 & 10.92 \\
Amargor & 7.25 & 8.89 & 9.13 & 8.21 \\
Consistencia & 9.45 & 9.32 & 9.04 & 9.64 \\
Sabor & 9.52 & 8.53 & & 9.01 \\
\hline
\end{tabular}

No existen diferencias significativas con error de $5 \%$. 
en cuanto a los parámetros $L^{*}$ y $b^{*}$, ya que en refrigeración la pasta posee menor luminosidad y menor color amarillo $(p<0,5)$.

La rancidez se consideró ligera, sin presentar diferencias al finalizar el almacenamiento; no obstante, el índice de peróxido aumentó significativamente al cabo de tres meses. La rancidez es percibida por el aroma y sabor del alimento (Jellinek, 1985) junto con otros componentes, que naturalmente han enmascarado la rancidez.

Los parámetros mejor calificados fueron apariencia y consistencia. El sabor fue característico a aceitunas sin llegar a ser excesivo, por lo que resulta ideal para ser utilizado como pasta untable.

\section{CONCLUSIONES}

Fue posible desarrollar una pasta de aceituna inocua hasta los 90 días de almacenamiento, con bajo $\mathrm{pH}$ y alta aceptabilidad sensorial. Ambas temperaturas de almacenamiento $-18^{\circ}$ y $4{ }^{\circ} \mathrm{C}$ - son aptas para la conservación de la pasta untable de aceitunas, ya que se mantienen las características, pero es más apropiado el almacenamiento en refrigeración porque el índice de peróxido alcanza menores valores en esta condición.

\section{REFERENCIAS}

A.O.A.C, 1995. Official Methods of Análysis of the Association of Official Analytical Chemist International. Volumes I y II. $16^{\text {th }}$ edition. Maryland, U.S.A.

A.O.C.S. 1993. Oficial Methods and Recommended Practices of the American Oil Chemists'Society. $3^{\text {th }}$ Edition. Champaign, Illinois.

Acín S, Navarro MA, Perona JS, Arbonés-Mainar JM, Surra JC, Guzmán MA, Carnicer R, Arnal C, Orman I, Segovia JC, Osada J, Ruiz-Gutiérrez V. 2007. Olive oil preparation determines the atherosclerotic protection in apolipoprotein E knockout mice. J. Nutr. Biochem. 18, 418-424.

Anzaldúa-Morales A. 1994. La evaluación sensorial de los alimentos en la teoría y la práctica. Editorial Acribia, España.

Berry EM, Eisenberg S, Friedlander $Y$, Harats D, Kaufmann NA , Norman Y, Stein Y. 1992. Effects of diets rich in monounsaturated fatty acids on plasma lipoproteins: the Jerusalem Nutrition Study. II. Monounsaturated fatty acids vs carbohydrates. Am. J. Clin. Nutr. 56, 394-403.

Bouaziz M, Chamkha M, Sayadi S. 2004. Comparative study on phenolic content and antioxidant activity during maturation of the olive cultivar chemlali from Tunisia. J. Agric. Food Chem. 52, 5476-5481.

Calíbrese G. 2002. Efectos del aceite de oliva virgen extra beneficios para la salud. Olivae 93, 19-20.

Callejas R, Barraza J, Kania A. 2001. Evaluación durante la cosecha de los principales factores que provocan el rechazo de aceitunas tipo verde sevillana destinadas al mercado extranjero. V Jornadas Olivícolas Nacionales. Vallenar, Chile. 79-81.

Callejas R, Pastene C. 2000. Algunos aspectos críticos del cultivo del olivo en el Valle del Huasco, $3^{\underline{a}}$ región. Aconex 68, 27-32.
Chikthimmah N, Laborde LF, Beelman RB. 2003. Critical factors affecting the destruction of Escherichia coli 0157:H7 in apple cider treated with fumaric acid and sodium benzoate. Food Microbiol. Safety 68, 14381442.

Conforti F, Sosa S, Marrelli M, Menichini F, Statti GA., Uzunov D, Tubaro A, Menichini F. 2009. The protective ability of Mediterranean dietary plants against the oxidative damage: The role of radical oxygen species in inflammation and the polyphenol, flavonoid and sterol contents. Food Chem. 112, 587-594

Consejo Oleícola Internacional. 2002. Norma cualitativa unificada applicable a las aceitunas de mesa en el comercio internacional. En: Legislación y normas sobre el aceite de oliva y las aceitunas de mesa. Ediciones Mundi-Prensa. Madrid, España.

Eisenberg, S. 2007. Relative Stability of Selenites and Selenates in Feed Premixes as a Function of Water Activity. JAOAC Internacional. 90, 349-353.

F.A.O. 1991. Elaboración de aceitunas de mesa. Organización de las Naciones Unidas para la Agricultura y la Alimentación. Sevilla, España.

Fernández M, De Castro Y Ramos, Garrido A, González CF, González PF, Nosti M, Heredia A, Mínguez M, Rejano L, Durán M, Sánchez F, García P, Castro. 1985. Biotecnología de la aceituna de mesa. Instituto de la Grasa y sus derivados. Sevilla, España.

F.I.A. 1999. El cultivo del olivo, diagnóstico y perspectivas. Fundación para la Innovación Agraria. Ministerio de Agricultura, Santiago, Chile.

F.I.A 2002. Estrategia de innovación agraria para producción olivícola. Fundación para la Innovación Agraria. Ministerio de Agricultura, Santiago, Chile.

García JM, Gutiérrez F, Barrera MJ, Alvi MA. 1996ª. Storage of mill olives on an industrial scale. J. Agric. Food Chem. 44, 590-593.

García JM, Gutiérrez F, Castellano JM, Perdiguero S, Morilla A, Albi MA. 1996 ${ }^{\mathrm{b}}$. Influence of storage temperature on fruit ripening and olive oil quality. $J$. Agric. Food Chem. 44, 264-267.

García P, Brenes M, Romero C, Garrido A. 1999. Color and textura of acidified ripe olives in pouches. J. Food Sci. 64, 248-251.

Gotoh N, Wada S. 2006. The importance of peroxide value in assessing food quality and food safety. JAOCS 83, 473-474.

Gutiérrez F, Perdiguero S, García JM, Castellano JM. 1992. Quality of oils from olives stored under controlled atmosphere. JAOCS 69, 1215-1218.

Hargrove RL, Etherton TD, Pearson TA, Harrison EH, Kris-Etherton PM. 2001. Low fat and high monounsaturated fat diets decrease human low density lipoprotein oxidative susceptibility in vitro. J. Nutr. 131, 1758-1763.

Hermoso M, Uceda M, Frias L, Beltrán G. 1998. Maduración. En: El cultivo del olivo. Ediciones MundiPrensa. Madrid, España.

Jacotot B. 2001. Interés nutricional del aceite de oliva. Olivae 86, 27-29.

Jellinek G. 1985. Sensory evaluation of Food. Ellis Horwood Ltd. Chichester, England.

Kalua C M, Allen M S, Bedgood Jr D R, Bishop A, Prenzler P, Robards K. 2007. Olive oil volatile compounds, flavour development and quality: a critical review Food Chem. 100, 273-286.

Kalua C, Bedgood D, Bishop A, Prenzler P. 2008. Changes in virgen olive oil quality during lowtemperature fruit storage. J. Agric. Food Chem. 56, 2415-2422. 
Marsillo V. 2002. Análisis sensorial de las aceitunas de mesa. Olivae 90, 32-41.

Martínez de Victoria E, Mañas M. 1998. El aceite de oliva en la dieta y la salud humana. En: El cultivo del olivo. Madrid, España. Ediciones Mundi-Prensa.

Mata P, Varela O, Alonso R, Lahoz C, De Oya M, Badimon L. 1997. Monounsaturated and polyunsaturated $n-6$ fatty acid-enriched diets modify LDL oxidation and decrease human coronary smooth muscle cell DNA synthesis, Arterioscler Thromb. Vasc. Biol. 17, 20882095

Mazzobre M, Schebor C, Burin L, Chirife J. 2002. Relevamiento del pH y la actividad de agua de conservas "en escabeche" y otras similares de elaboración artesanal/doméstica en relación al riesgo potencial para el crecimiento de Clostridium botulinum. Departamento de Industrias, Facultad de Ciencias Exactas y Naturales, Universidad de Buenos Aires, Argentina. Disponible en el http://www.nutrinfo.com.ar.

Ministerio de Salud. 1998. Reglamento Sanitario de Alimentos . Santiago, Chile.

NCh 2734.Of2002. Determinación de hongos y levaduras - Técnica de recuento en placa. Instituto Nacional de Normalización. Santiago, Chile.

NCh 2659.of2002. Determinación de microorganismos aerobios mesófilos - Técnica de recuento en placa a $35^{\circ} \mathrm{C}$. Instituto Nacional de Normalización. Santiago, Chile.

NCh 2676.of2002. Determinación de Enterobacteriaceae sin resucitación - Técnica NMP y técnica de recuento en placa. Instituto Nacional de Normalización. Santiago, Chile.

ODEPA. 2009. Oficina de Estudios y Política Agraria. Gobierno de Chile http://www.odepa.gob.cl/odepaweb/ jsp/odepad.jsp

Prochile. 2009. www.prochile.cl

Sánchez AH, Montaño A, Rejano L. 1997. Effect of preservation treatment, Light and storage time on quality parameters of Spanish - style green olives. J. Agric. Food Chem. 45, 3881-3886.
Schmidt-Hebbel H, Pennacchiotti I, Masson L, Mella M A. 1992. Tabla de composición química de alimentos chilenos. Facultad de Ciencias Químicas y Farmacéuticas. Departamento de Ciencias de los Alimentos y Tecnología Química. Universidad de Chile. Octava edición. Edición Digital reproducida con autorización del autor.

Schwartz M, Kern W, Marchant R, Callejas R, Sepúlveda M. 2001. Actualización del diagnóstico del sector olivícola nacional y formulación de estrategias de desarrollo. Parte 1. Aceituna de mesa. Ministerio de Relaciones Exteriores, Dirección General de Relaciones Económicas Internacionales, Dirección de promoción de Exportaciones (Pro Chile).

Tortora G, Berdell R, Case C. 1993. Introducción a la microbiología. Editorial Acribia S. A. Zaragoza, España.

Turanta F, Göksungur Y, Dinçer H, Ünlütürk A, Güvenç U, Zorlu N. 1999. Effect of potassium sorbate and sodium benzoate on microbial population and fermentation of black olives. J. Sci. Food Agriculture 79, 1197-12

Ünal K, Nergiz C. 2003. The effect of table olive preparing methods and storage on the composition and nutritive value of olive. Grasas y Aceites. 54, 71-76.

USDA National Nutrient Database for Standard Reference. www.nal.usda.gov/fnic/foodcomp/Data/S R17/wtrank/sr17w601.pdf).

Venegas N, Marambio E, Insulza M, Soto A, Arrieta A. 1990. Control microbiano de alimentos; Técnicas actualizadas y métodos acelerados. Publicaciones Miscelaneas Agrícolas $\mathrm{N}^{\circ}$ 32. Departamento de Agroindustria y Tecnología de alimentos, Facultad de Ciencias Agrarias y Forestales, Universidad de Chile.

Wittig E. 2001. Evaluación Sensorial. Una metodología actual para tecnología de alimentos. Edición Digital reproducida con autorización del autor.

Recibido: $2 / 2 / 09$ Aceptado: 10/3/09 